\title{
Modeling Students' Emotions from Cognitive Appraisal in Educational Games
}

\author{
Cristina Conati, Xiaoming Zhou \\ Department of Computer Science, University of British Columbia, Vancouver, BC, Canada, \\ \{conati, xzhou\}@cs.ubc.ca
}

\begin{abstract}
We present a probabilistic model that assesses student emotional reaction during interaction with an educational game. Following a well-known cognitive theory of emotions (the OCC theory), the model predicts a student's emotional state by assessing the student's appraisal of her interaction with the game, in light of the student's goals and personality. We illustrate how the model relies on a Dynamic Decision Network that is based on both the OCC theory and observations from two user studies.
\end{abstract}

\section{Introduction}

Learner motivation is a key component for the success of any pedagogical activity. Electronic games for education are learning environments that try to increase the learner's motivation by embedding pedagogical activities in highly engaging, gamelike interactions. However, several studies show that, while these educational games are usually successful in increasing student engagement, they often fail in triggering learning [7]. The studies indicate that this happens because many students play the games without actively reasoning about the underlying instructional domain, and thus fail to learn from the game activities.

To overcome this limitation of educational games, we are designing pedagogical agents that, as part of game playing, generate tailored interventions aimed at stimulating the student to learn better from the game. However, in order not to interfere with the high level of engagement that is a key asset of educational games, these agents need to take into account the players' affective states in addition to their cognitive states when deciding how to act.

Toward this end, we are devising a probabilistic model of student affect that our pedagogical agents can use, along with an assessment of student learning, to generate interventions that improve learning without compromising engagement. The model relies on a Dynamic Decision Network (DDN) [9] to probabilistically integrate information on both the possible causes of affective reaction and its observable effects (e.g., a change in the student's facial expression). Leveraging any evidence available on the student's emotional state is crucial, because in this modeling task the different sources of evidence are often ambiguous, and vary significantly with both the student and each particular interaction.

In this paper, we focus on the part of the model that reasons about the causes of student's affect by relying on the OCC cognitive theory of emotions, developed by Orthony, Clore and Collins [8]. The OCC theory describes a person's emotions as the result of that person's appraisal of how the current situation fits with the person's goals and preferences. Our DDN models a student's appraisal mechanism during interaction with an educational game. It does so by representing how game events 
relate to the students' goals, as well as how these goals probabilistically depend on the students' traits and playing behavior.

Because affective user modeling is a rather new research area, only a few computational modes of user affect have been devised to date. A model that, like ours, is based on the OCC theory is discussed in [4]. This model is not probabilistic, and does not specify how to identify users' goals, traits and preferences. A model that uses fuzzy rules to assess anxiety in combat pilots is described in [5]. Because of the specificity of the modeling task, the model does not need to deal with the high level of uncertainty involved in modeling affect in less constraining interactions, like those generated by educational games. The affective model proposed in [1] relies on a probabilistic approach, but it uses only evidence on the effects of emotional arousal, not on its causes. For this reason, the model can assess only intensity and valence (i.e., whether the emotion is positive or negative) of the user's emotional states. [6] discusses how eyebrow movements and posture can provide partial evidence to infer a student's engagement while interacting with a computer-based tutor.

In the rest of the paper, we first present the educational game that we currently use as a test bed for our research. We then describe how our model was built by integrating the OCC cognitive theory of emotions with observations we collected in two user studies. We conclude with a discussion of future work.

\section{The Prime Climb Educational Game}

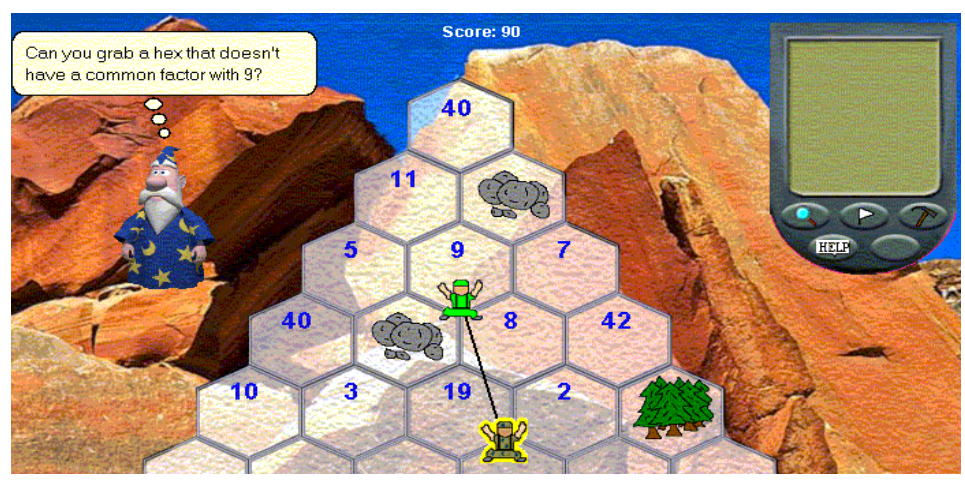

Figure 1: Screenshot of the Prime Climb interface

Prime Climb is a game designed by the EGEMS (Electronic Games for Education in Math and Science) group at the University of British Columbia to help students learn number factorization. In Prime Climb, two players must climb ice-faces divided in numbered sections (see Figure 1). Each player can only move to sections with numbers that do not share any factors with that occupied by the other player. A player who makes a wrong move falls and the climbing team looses points. For instance, the player at the bottom in Figure 1 fell because she moved to section 42, which shares factor 3 with section 9, where the other player is. To help the students understand factorization, Prime Climb includes tools to inspect the factorization of the numbers on the mountain, accessible via the PDA at the top-right corner of Figure 1. 
Before climbing a mountain with a peer, each player must get certified by doing practice climbs with an instructor. This instructor is one of the intelligent agents that we are designing to improve the pedagogical effectiveness of Prime Climb. The function of this agent is to be the student's climbing partner during the practice climbs and provide hints (both unsolicited and on demand) that help the student to reason about number factorization, without compromising her level of engagement. These hints include (i) making the student think about the reasons for a fall, and (ii) giving specific advice on how to avoid or recover from a fall (see Figure 1, on the left). The affective model that we describe in the rest of the paper is devised to assess student affect during the interaction with the climbing instructor. The model is used by the instructor (the agent, from now on) to decide when and how to provide help. We chose to model student affect during the practice climb phase of the game to test our approach on a simpler scenario before extending it to the more complex task of modeling student affect during the climbs with a peer.

\section{A Dynamic Decision Network to Model Student Affect}

Modeling student affect during the interaction with an educational game is a task frequently permeated with uncertainty. One cause for this uncertainty is that often the same situation can induce a variety of different emotional states in different students, depending upon student properties that are not always easily observable (e.g., goals, preferences, personality). A second cause of uncertainty is that the bodily expressions that are symptoms of emotional arousal (e.g., changes in facial expressions, increased heart rate) can be difficult to assess precisely and seldom support a one-to-one mapping with emotional states.

To handle the high level of uncertainty in this modeling task, we explicitly represent the probabilistic nature of the relations between student emotional states, their causes and effects using Dynamic Decision Networks (DDNs) [9]. DDNs are an extension of Bayesian networks [9] that can model, in addition to static random variables, (i) an agent's deliberate actions, represented as decision variables; (ii) the agent's preferences over the possible outcomes of the actions; and, (iii) the evolution of variables over time. The advantage of a model based on DDN is that it can leverage any evidence available on the variables related to emotional states to make predictions for any other variable in the model. For instance, we can assess both emotional states and personality traits from bodily expressions (i.e., the effects of the emotions), if a sufficient number of bodily expressions can be reliably observed (see diagnostic assessment in Figure 2). Or, the student's emotional state can be assessed using existing information on relevant student's traits and the current situation (i.e., the causes of emotions), even if there is little evidence on bodily expressions (see predictive assessment in Figure 2). Furthermore, DDNs provide algorithms that can compute, at any decision point, the agent action with the maximum expected utility, thus providing a decision theoretic basis for the agent behavior.

In this paper, we focus on the part of the model that performs predictive assessment (more details on the diagnostic part can be found in [2]). Figure 2 shows two time slices of the DDN that forms our model of student's affect. The nodes in Figure 2 represent classes of variables in the actual DDN. The part of the network above the nodes Emotional States represents the relations between possible causes and emotional 


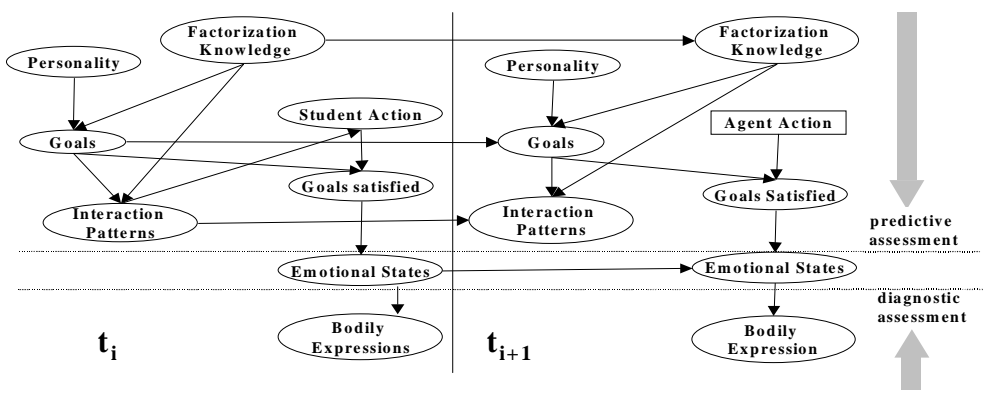

Figure 2: Two time slices of the DDN to model student affect

states, as they are described in the OCC cognitive theory of emotions devised by Ortony, Clore and Collins [8]. In this theory, emotions arise from valenced (positive or negative) reactions to situations consisting of events, actors and objects. The valence of one's emotional reaction depends upon the desirability of the situation for oneself, which in turn is defined by one's goals and preferences. For instance, an event that matches one's goal can generate positive emotions such as joy for the event, admiration toward the actor that caused the event, or pride if the actor is oneself. Corresponding negative emotions for an event that interferes with one's goals are distress, reproach and shame.

To apply the OCC theory to the assessment of emotions in Prime Climb players, our DDN includes variables for goals that students may have when playing the game, summarized in Figure 2 by the nodes Goals ${ }^{l}$. The object of the student's appraisal is any event caused by either a student's game action (node Student Action in Figure 2, time slice $\mathrm{t}_{\mathrm{i}}$ ) or an agent's action. Agent actions are represented as decision variables in the model (e.g., the rectangular node Agent Action in Figure 2, slice $\mathrm{t}_{\mathrm{i}+1}$ ) indicating points in which the agent makes choices about if and how to intervene in the game $e^{2}$. The desirability of an event in relation of the student's goals is represented by the nodes class Goals Satisfied, which in turn influences the student's emotional state.

User's goals are a key element of the OCC model, but assessing these goals is not trivial, especially when asking the user directly is not an option, as is the case in educational games. Thus, our DDN also includes nodes that can help the model infer the student's goals from indirect evidence. What goals a student has can depend on both the student's Personality [3] and Factorization Knowledge, as represented by the links connecting the corresponding nodes with the Goals node in Figure 2. Also, the student's goals can influence how a student plays the game, as this influence is represented in the DDN by the link between the nodes Goals and Interaction Patterns in Figure 2. In turn, interaction patterns can be inferred incrementally from specific features of the student's individual actions at each time slice. Thus, observations of both the relevant student's traits and game actions can provide the DDN with indirect evidence for assessing the student's goals.

In Figure 2, the links between nodes in different time slices indicate that the values of the corresponding variables evolve over time and that their value at time $t_{i}$ influences

\footnotetext{
${ }^{1}$ We currently do not explicitly represent player preferences in our model.

${ }^{2}$ Due to space constraints, we omit the description of how the model is used to inform the agent's choice.
} 
the value at time $t_{i+1}$. These links model, for example, the fact that a student is more likely to feel a given emotion at time $t_{i+1}$ if the student felt it at time $t_{i}$. A new time slice is added to the network when either the student or the agent performs an action. However, only two time slices are maintained at any give time, because the influence of previous time slices can be summarized as prior probabilities in the first of the two active slices (e.g., slice $t_{i}$ in Figure 2). We now describe in more detail the nodes that allow predicting student emotions from situation appraisal.

\section{Nodes and Structure of the Appraisal Model}

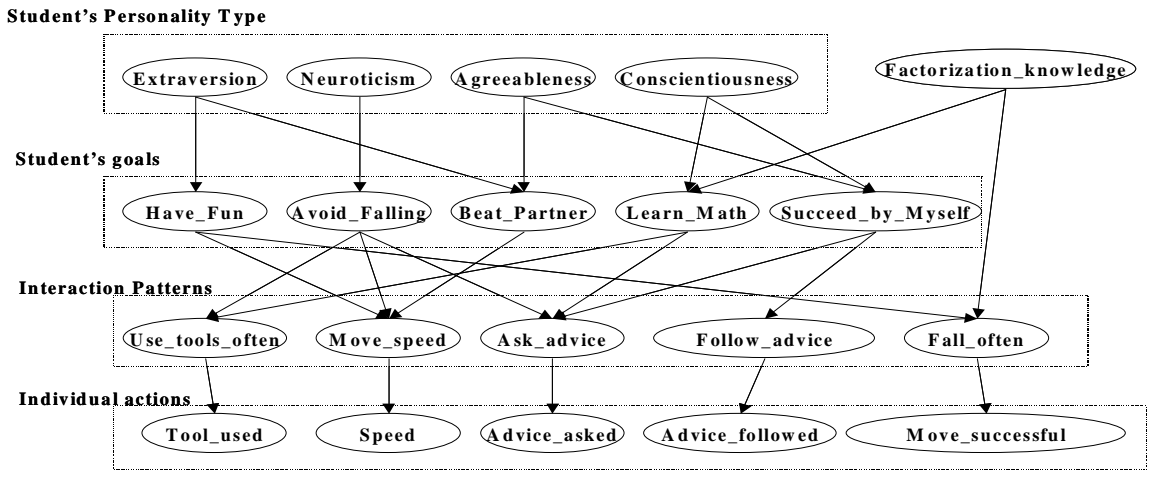

Figure 3: Student traits, goals and moving patterns in one time-slice of the DDN

The elements of the appraisal model that do not derive directly from the OCC theory are the result of combining other relevant findings from psychology with our intuition and with observations of students playing Prime Climb. These observations were collected from two Wizard of $\mathrm{Oz}$ studies, involving 23 students who engaged in practice climbs with the climbing instructor agent. Students could ask the agent for help at any time by clicking on a help button available in the interface. An experimenter (Wizard) controlled the agent from a computer placed in a separate room, and provided help both unsolicited and on-demand.

Before playing with Prime Climb, students were given a pre-test to evaluate their knowledge of number factorization. Afterward, they filled out a questionnaire probing, among other things, what high level goals students had while playing the game. In both studies, an observer recorded students' behaviors. In the second study (involving 10 of the 23 students) we also collected log files of the interactions.

Student goals. Our model currently includes 5 high level goals that students playing Prime Climb may have. As shown in Figure 3, these goals include Have Fun, Avoid Falling, Beat Partner, Learn Math and Succeed by Myself (i.e., without the agent's help). The goals Have Fun, Learn Math and Avoid Falling are plausible goals that students may establish given the nature of the game. The goal Succeed_by_Myself was clearly shown by some students who not only avoided asking the agent for help, but also looked annoyed when the agent volunteered it. The goal Beat Partner is inconsistent with the nature of the game, as climbing the Prime Climb mountain is designed to be a collaborative effort. However, 8 out of the 23 subjects in the Wizard 
of Oz studies selected this goal in the post-questionnaire (see Table 1), and the goal is consistent with findings indicating that certain personality types tend to be competitive even during collaborative interactions. The goal nodes in the DDN are currently Boolean, where a $\mathrm{T}$ value represents the probability that a player has the corresponding goal.

Table 1: number of students that selected each goal in the post-questionnaire

\begin{tabular}{|c|c|c|c|c|} 
Have Fun & Avoid Falling & Beat Partner & Learn Math & Succeed By Myself \\
\hline 14 & 15 & 8 & 12 & 8 \\
\hline
\end{tabular}

Student personality traits and math knowledge. Because personality is known to influence one's goals and behaviours [3], our model contains nodes and links representing student personality types and their relations to student goals in playing Prime Climb. Thus, when accurate priors are available for personality nodes, the DDN can use them as additional information to estimate the probability of goals, if goals cannot be directly established. Nodes and links for personality types have been chosen following the Five-Factor Model of personality [3]. In the Five-Factor Model, personality traits are structured as five domains - neuroticism, extraversion, openness, agreeableness and conscientiousness. As Figure 3 shows, our affective student model currently includes variables for only four of the five domains, because openness did not seem directly relevant to our task. The links modeling relationships between the four personality nodes and the student's goals are based on the definitions of the four domains in the Five-Factor Model. For example, an agreeable person is defined as "fundamentally altruistic.... eager to help...and believes that others will be equally helpful in return". By contrast, the disagreeable person is "egocentric, sceptical of others' intentions, and competitive rather cooperative." Thus, in our model, the conditional probabilities for the Beat-Partner and Succeed-by-Myself goals reflect the fact that they are likely for a disagreeable person, and unlikely for an agreeable one. Personality nodes currently have two values, representing the extremes of the corresponding personality types.

In addition to personality, another student trait that may influence student goals is knowledge of number factorization. This trait is represented in our DDN by the variable Factorization Knowledge in Figure 3, and influences the goal Learn_Math because of our intuition that having high math knowledge can make a student less interested in learning math through the game. However, further studies in which we have information on the subjects' personality are needed to accurately specify the dependencies between domain knowledge, personality and goals.

Interaction patterns. Another source of information that can be used to infer students' goals is how they play the game. Our data indicate several dependencies between students' goals and their playing behavior. For instance, we found that (i) a student who wants to succeed by herself is unlikely to ask the agent for advice; (ii) a student who has the goal to learn math is more likely to access the Prime Climb tools to inspect number factorization; (iii) students that have the goals Have_Fun and Beat_Partner are more likely to move quickly, while students that want to avoid falling are more likely to move slowly. As Figure 3 shows, our model currently includes variables for five interaction patterns that our data indicate to be directly 
linked to students' goals: Use_tools_often, Move_speed (indicating how quickly the student moves from one number to another), Ask_advice, Follows_advice, Fall_often. One of these interaction patterns, Fall_often, is influenced by both the goal Have_Fun and by the student's factorization knowledge. This allows the system to model the fact that a few students kept falling because they found it amusing. Thus, the model considers frequent falls as an indication of either lack of factorization knowledge or the goal to have fun.

Student individual actions. Evidence for interaction patterns is collected directly from individual student interface actions, described by the nodes at the bottom of Figure 3. Every time the student performs an action, a new time slice is created in the dynamic network, and the relevant nodes describing the action and its outcome are set as evidence. The node Tool_used is set every time the student activates one of the tools available to inspect number factorization. The outcome of a student's move (i.e. whether the student fell or not), provides evidence for the node Move_successful, while the speed of a move provides evidence for the node Speed. Advice_asked is set to true every time the student asks the agent for advice, while the value of Advice_followed depends upon whether or not the student followed the agent's advice. Some of the nodes describing student actions also provide evidence defining the situation used in the appraisal process (described later in this section). Two of these nodes are shown in Figure 4: Move_successful, described above, and Ahead-ofpartner, which is also instantiated after a student move and indicates whether or not the student is ahead of her partner after the move. The values of these nodes influence how the DDN models the student's appraisal of her action's outcome.

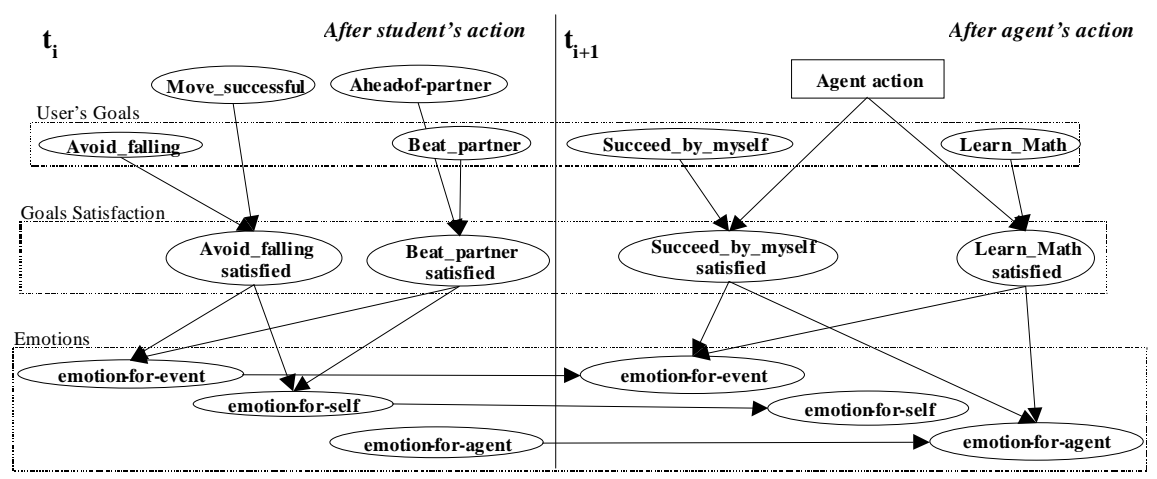

Figure 4: Sample of the nodes involved in action appraisal and emotion assessment

Agent actions. Agent actions are represented as a decision node in the DDN, (e.g., node agent action in Figure 4). Like student actions, each agent action adds a new slice to the DDN. We currently summarize the agent's actions as three different values of the decision node: (1) Hint (an indirect suggestion to help the student overcome a climbing impasse); (2) Move To (explicitly telling the student where to move); (3) Reflect (asking the student to reason about the outcome of a move).

Situation appraisal. The appraisal mechanism which dictates how the outcome of the student or agent actions relates to student goals is represented in our DDN by the 
links between goal nodes, action nodes and a new layer of nodes representing goal satisfaction (see Figure 4). For each goal node $g$ in the DDN, a corresponding Boolean node g_satisfied describes whether goal $g$ is satisfied after each agent or student action (Figure 4 shows a sample of the goal satisfaction nodes in the network). For example, if the student has the goal Avoid_Falling and makes a move at time $\mathrm{t}_{\mathrm{i}, \text { the }}$ value of the node Avoid_Falling_satisfied in that time slice depends upon whether the move is successful. Similarly, if the student has the goal Succeed_by_Myself, the probability of the goal Succeed_by_Myself_satisfied decreases if the agent gives general advice about how to climb, and decreases even more if the agent explicitly tells the student where to move. The values of the goal satisfaction nodes are the direct cause of the student's emotional states, as we describe next.

Student emotional states. While the OCC theory specifies 22 emotion types, we currently represent in our DDN only the 6 that are more directly relevant to assessing student engagement during the Prime Climb practice climbs. Two of these emotions, joy and distress, are directed toward the event that is the object of student appraisal. Two other emotions, pride and shame, are directed toward the student herself and arise when the student caused the appraised event. The other two, admiration and reproach, are directed toward the agent, when the agent caused the event. The three pairs of emotions are represented in the network by three corresponding two-valued nodes (see Figure 4), emotion_for_event, emotion_for_self and emotion_for_agent, because we currently assume that the emotions in each pair are mutually excusive and that each interaction event simply changes the probability that the student can be in either of the two emotional states. Further empirical validations may lead us to move to a model in which each emotion is represented by a different variable. Currently, the model does not include a separate node for the student's emotion toward the climbing partner because in this phase of the game the climbing partner is the agent.

In each time slice, all the goal satisfaction nodes are linked to the emotion_for_event node, to represent the result of event appraisal. In addition, goal satisfaction nodes are linked to the emotion_for_self node if the time slice includes a student's actions (see Figure 4, slice $\mathrm{t}_{\mathrm{i}}$ ), or to the emotion_for_agent node if the time slice includes an agent's action (see Figure 4, slice $t_{i+1}$ ). Links between emotion nodes across time slices model the evolution of emotions over time. In particular, the conditional probabilities for an emotion node with no parent goal satisfaction node (e.g., emotion-for-self in Figure 4) model the fact that an emotion fades over time if no event revives it.

Keeping the distinction between the student's emotions toward self and toward the agent is important to help the agent decide how to act. For example, if the probability of shame is high the agent can decide to provide hints aimed at making the student feel better about her performance, while if the probability of reproach is high the agent needs to take actions that allow it to regain credibility.

\section{Sample Assessment}

To exemplify the workings of our model, we illustrate the assessment it generates during a simple simulated interaction. In this interaction, the student (i) moves quickly, (ii) never falls and (iii) is often ahead of the partner. Four times during the 
interaction (marked by the asterisks on the bottom axis in Figure 5), the agent asks the student to reflect on the outcome of a move and the student ignores the advice.

Suppose that we don't have any knowledge of the student's personality. Since the student repeatedly ignores the agent's advice, the probability of the interaction pattern Follow_advice decreases, and that of the goal Succeed_by_Myself consequently increases (see Figure 5, bottom chart). The probability of the goals Have_Fun and Beat_Partner also increases, because both goals receive evidence from the fact that the student moves quickly (see Figure 3). The middle chart in Figure 5 shows the corresponding assessment of the student's emotional state. The probability of reproach increases slightly every time the agent intervenes, because the intervention interferes with the goal Succeed_by_Myself; then it slowly decreases over the time slices when the agent lets the student be. Despite the four agent interventions, the goal Succeed_by_myself is satisfied in the majority of the time slices because the student always makes successful moves without following the agent's advice. The goal Beat_Partner is also likely to be satisfied, because the player stays mostly ahead of the partner. Thus, the probability of pride constantly increases, while the probability of joy increases in all but those slices corresponding to the agent's interventions.

Changes in the probabilities of goal nodes also propagate upward to influence the assessment of the student's personality. The probability of the personality type Disagreeablenes increases because it is supported by both goals Succeed_by_Myself

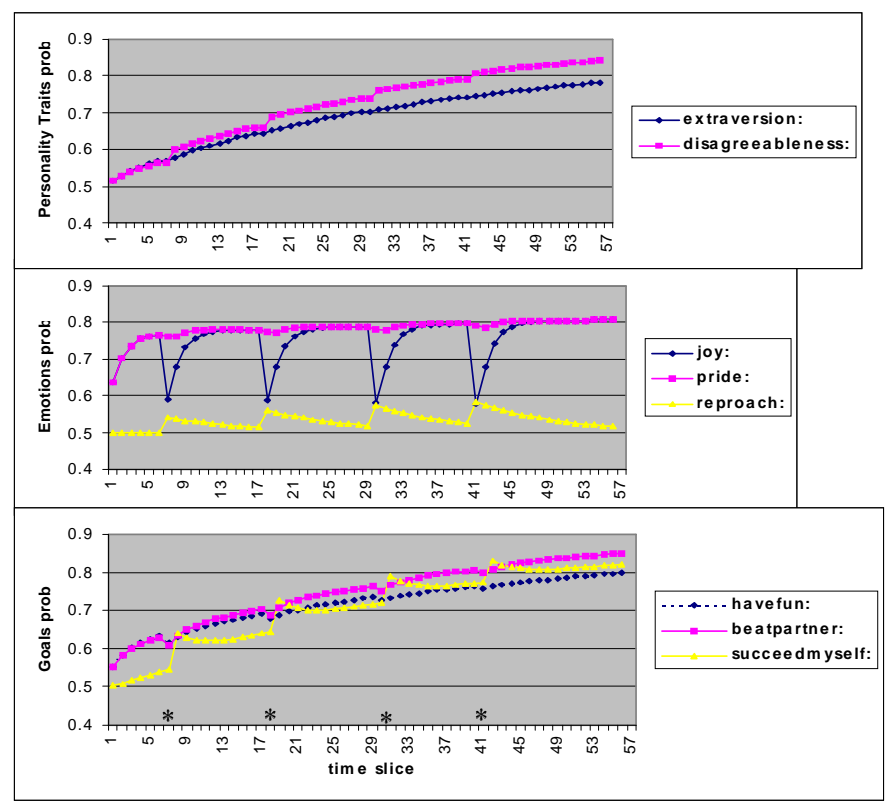

Figure 5: Sample assessment of goals, emotions and personality

and Beat_Partner, while the probability of the personality type Extraversion increases because of the support coming from the goal Have_Fun (see top chart in Figure 5).

As we mentioned at the beginning of the paper, the assessment of the affective model should be combined with an assessment of student learning to enable the agent to 
improve this learning without compromising student engagement. Consider, for instance, the example described above. The affective model clearly indicates that the student feels reproach for the agent when it tries to make the student reason about her moves. This suggests that the agent should avoid intervening in the student's playing. However, the four agent's interventions in this example might be justified if the agent had some reason to believe that some of the student's successful moves were not due to a real understanding of the factorization of the numbers involved. The agent might also have decided that causing the student's reproach wouldn't seriously compromise her engagement, because the high probability of joy and pride indicates that overall the student is enjoying the game. Finally, recognizing that the student most likely has the goal of having fun, the agent might try to compensate for its pedagogical interventions by generating actions that have the sole purpose of amusing the student.

\section{Conclusions and Future Work}

We have presented a probabilistic model that relies on a Dynamic Decision Network to assess student emotions during the interaction with an educational game. The model was built by integrating the OCC cognitive theory of emotions with data gathered during two user studies. It assesses affect by predicting how student goals, personality and knowledge influence student appraisal of the interaction with the game.

Several are the directions for future work. First, we will refine the model by running additional studies with students for which we have a personality assessment. Third, we will integrate the affective model with a model of student learning, and define the utility functions that will guide the selection of agent actions in the DDN. Lastly, we will extend the model to assess student affect during the climb with a peer.

\section{References}

1. Ball, G. and J. Breese. Modeling the emotional state of computer users. in Workshop on 'Attitude, Personality and Emotions in User-Adapted Interaction', UM '99, 1999.

2. Conati, C. Probabilistic Assessment of User' s Emotions During the Interaction wht Educational Games. To appear in Journal of Applied Artificial Intelligence.

3. Costa, P.T. and R.R. McCrae, Four ways five factors are basic. Personality and Individual Differences. 1992. 13(1) p. 653-665.

4. Elliott, C., J. Rickel, and J. Lester,. Lifelike Pedagogical Agents and Affective Computing: An Exploratory Synthesis. Artificial Intelligence Today, Lecture Notes in Computer Science 1600, M. Wooldridge and M. Veloso (eds.), 1999. Springer Verlag. 195-212.

5. Hudlicka, E. and D. McNeese. Assessment of User Affective and Belief States for Interface Adaptation: Application to an Air Force Pilot Task.. Journal of User Modeling and UserAdapted Interaction, 2002. 12(1): p. 1-47.

6. Kaapor, A., S. Mota, and R. Picard. Toward a learning companion that recognizes affect. in AAAI Fall Symposium: 'motional and Intelligent II', 2001. AAAI Press.

7. Klawe, M. When Does The Use Of Computer Games And Other Interactive Multimedia Software Help Students Learn Mathematics? in NCTM Standards 2000 Technology Conference, 1998. Arlington, VA.

8. Orthony, A., G.L. Clore, and A. Collins. The cognitive structure of emotions, 1988. Cambridge University Press.

9. Russell, S. and P. Norvig. Artificial Intelligence: A Modern Approach, 1995. MorganKaufman. 